\title{
L'itinéraire d'un aristocrate au service des "arts utiles" : Servières, alias Reth (1755-1804)
}

The itinerary of an aristocrat devoted to the "useful arts ": Servières, alias Reth (1755-1804)

\section{Christiane Demeulenaere-Douyère}

\section{OpenEdition Journals}

Édition électronique

URL : http://journals.openedition.org/dht/1010

DOI : 10.4000/dht.1010

ISSN : 1775-4194

\section{Éditeur :}

Centre d'histoire des techniques et de l'environnement du Cnam (CDHTE-Cnam), Société des élèves du CDHTE-Cnam

\section{Édition imprimée}

Date de publication : 1 juin 2008

Pagination : 64-76

ISBN : 978-2-95-30779-1-9

ISSN : 0417-8726

\section{Référence électronique}

Christiane Demeulenaere-Douyère, "L'itinéraire d'un aristocrate au service des "arts utiles" : Servières, alias Reth (1755-1804) », Documents pour l'histoire des techniques [En ligne], 15 | $1^{\mathrm{er}}$ semestre 2008, mis en ligne le 21 octobre 2010, consulté le 07 septembre 2020. URL : http://journals.openedition.org/dht/ 1010 ; DOI : https://doi.org/10.4000/dht.1010 


\title{
L'itinéraire d'un aristocrate au service des " arts utiles" : Servières, alias Reth (1755-1804)
}

\author{
Christiane Demeulenaere-Douyère \\ Archives nationales (site de Paris) \\ CDHTE-Cnam
}

\begin{abstract}
RÉSUMÉ
En 1791, inventions et inventeurs se retrouvèrent au centre d'une activité législative considérable. Soucieux de s'émanciper des procédures de validation de l'Ancien Régime, les inventeurs obtinrent le vote de lois garantissant leur propriété sur leurs découvertes et la mise en place, avec le Bureau de consultation des arts et métiers, d'un système officiel de reconnaissance et d'encouragement. En qualité de président de la Société des inventions et découvertes, société libre d'artistes-inventeurs, peut-être même son fondateur, Claude-Urbain de Retz de Servières (1755-1804) a joué un rôle important dans l'adoption en France de cette législation, et il a de ce fait été un acteur de l'institutionnalisation de l'invention. Pourtant, cet aristocrate en rupture avec son milieu d'origine, converti aux idées républicaines, qui a poursuivi, sous le nom de Reth, sa carrière dans l'administration des Assignats, puis dans celle de la Loterie nationale, n'est guère connu pour le rôle qu'il a joué dans l'histoire de l'invention en France. II mérite mieux que ce semi-incognito.
\end{abstract}

Résumés et mots clés en anglais sont regroupés en fin de volume, accompagnés des mots clés français.

$E^{n}$ n histoire des techniques comme en histoire générale, il est des personnages dont le nom semble a priori familier, même si, à la réflexion, il n'est guère possible d'avancer plus de deux ou trois informations étayées les concernant.

Tel est le cas, par exemple, de Servières, que l'on connaît comme un des concurrents malheureux du général Bonaparte lors de l'élection de ce dernier à l'Institut national. Au-delà, on rencontre son nom parmi les correspondants de Lavoisier, et aussi parmi les membres du Bureau de consultation des arts et métiers. Telles étaient les quelques indications que nous possédions lorsque nous avons rencontré ce personnage à la tête de la Société des inventions et découvertes' ${ }^{1}$.

Les recherches que nous avons alors entreprises à son sujet ont révélé une personnalité et un itinéraire

1 Demeulenaere-Douyère $C$., « Inventeurs en Révolution : la Société des inventions et découvertes », Documents pour l'histoire des techniques, n 17, 2009, à paraître. qui ne manquent ni d'intérêt ni d'originalité, et qui méritent qu'on prenne le temps de s'y attarder.

Premier président de cette société d'inventeurs, peut-être même son fondateur (il figure parmi les signataires de l'acte de constitution de la Société en janvier 1791 en tant que « président provisoire »), il a joué, à ce titre, un rôle tout à fait considérable dans les négociations qui ont entouré l'élaboration d'une législation protectrice de l'invention, et a de ce fait été un acteur de l'institutionnalisation de l'invention en France.

\section{Une vieille famille aristocratique}

Claude-Urbain de Retz (ou de Reth²) de Bressolles, baron de Servières, naît à Mende (Lozère), le 10 janvier 1755³. II est I'aîné des dix enfants d'Urbain de Retz de

2 La prononciation anglaise du th de Reth est restituée approximativement en Retz.

3 Archives départementales (ensuite AD) Lozère, E dépôt Mende 
Servières d'Altier, seigneur de Servières et de Cougoussac, ancien capitaine au régiment de Chartres-Cavalerie, et de Jacquette de Moure de Villeret, son épouse. Authentique aristocrate, il appartient à une vieille famille noble du Gévaudan qui descend d'un archer de la Garde écossaise de Charles VII fixé en France au milieu du $x^{\mathrm{e}}$ siècle ${ }^{4}$.

Après des études au collège de Mende où il est le condisciple de Chaptal, il se destine à la carrière des armes, comme la plupart des fils aînés de familles nobles. La tradition familiale l'oriente, non pas vers un corps technique comme l'artillerie - ce qui aurait été plus en accord avec la suite de son itinéraire -, mais vers la cavalerie. L'ancienneté et la qualité de sa noblesse, ainsi que les relations paternelles, lui permettent d'être admis, en juin 1770, aux pages de la Grande Écurie du roi, à Versailles. C'est une position prestigieuse, mais dispendieuse pour sa famille (son père a parfois du mal à payer régulièrement sa pension), et surtout, dans le cas de Claude-Urbain de Retz de Servières, c'est une mesure disciplinaire qui sanctionne des débordements juvéniles: en mars 1770, le jeune homme a été en effet brièvement emprisonné à Mende, avec un de ses cousins, pour avoir troublé l'ordre public en tirant des coups de pistolet dans la rue, à l'occasion de l'installation de son père comme premier consul de la ville ${ }^{5}$.

À l'issue de son stage à la Grande Écurie, il obtient le rang de sous-lieutenant sans appointements (1773), puis passe sous-lieutenant (1774) dans le régiment d'Orléans-Cavalerie ${ }^{6}$. II le quittera, sans qu'on puisse préciser exactement quand, entre 1779, date à laquelle il se présente à la Société de l'abbé Baudeau comme officier de cavalerie, et 1785, date à laquelle il est qualifié $d$ ' « ancien officier ».

GG 72 .

4 Lescure M. H. F. C. de, Armorial du Gévaudan, Lyon, A. Badiou-Amant, 1929.

5 AD Lozère, 1 J 130. Je remercie ici vivement Mme Claire Martin, alors directrice des Archives de la Lozère, pour son aide précieuse concernant divers aspects de la jeunesse de Claude-Urbain de Retz de Servières en Gévaudan.

6 Sur son séjour aux pages de la Grande Écurie du roi, Archives nationales (ensuite AN) Paris, O/1*/954, 955, 959 (21), 965 et 969 et $\mathrm{O} / 1 / 973$; pour la suite de sa carrière militaire, Service historique des armées, 1 Yd 212 et Yg 569 : il obtient le rang de sous-lieutenant sans appointements le 31 janvier 1774 dans le régiment de cavalerie d'Orléans; nommé sous-lieutenant le 3 mai 1775, il est réformé en 1776 à l'occasion de la nouvelle organisation de la cavalerie et redevient sous-lieutenant le $1^{\text {er juin }}$ 1777 ; en 1776, il est noté « appliqué, bon sujet ».
Comme beaucoup d'officiers de son temps', le désœuvrement de la vie militaire aidant, Servières se forme à l'esprit des Lumières et s'adonne avec passion aux sciences. C'est un lecteur assidu du Journal de physique de l'abbé Rozier ${ }^{8}$, des diverses publications de l'Académie royale des sciences et des académies de province, et de très nombreux auteurs, depuis les Anciens comme Pline jusqu'aux plus contemporains. Ces lectures lui permettent de se constituer une culture scientifique assez éclectique ou tout au moins développent-elles chez lui une curiosité affirmée pour les phénomènes de la nature.

S'en font l'écho les observations et réflexions diverses qu'il publie lui-même très régulièrement dans le Journal de physique. Ces communications, si elles montrent une belle assurance - l'une d'elles commence en ces termes: " S'il est permis à un jeune physicien de faire part au public de ses idées ${ }^{9} \gg . .$. - , témoignent aussi d'une certaine érudition et d'une assez large ouverture d'esprit. Toutes les disciplines retiennent son intérêt : physique, histoire naturelle, minéralogie, paléontologie, arts et métiers... Mais il les aborde davantage en dilettante qu'en véritable scientifique et privilégie généralement l'observation et la description des objets et des faits à leur analyse expérimentale et à leur explication.

Servières est un homme de son temps ; curieux de tout, il s'essaie aussi à l'agronomie. On le voit s'informer auprès de la Société libre d'émulation de l'abbé Baudeau, en 1779, du programme du prix sur la distillation des marcs ${ }^{10}$. En 1780, dans une lettre à Benjamin Franklin, il se dit chargé par Necker de « composer un traité raisonné sur la culture du tabac ${ }^{11} »$. Il publie plusieurs mémoires sur la culture de l'ortie et « ses usages

7 On pense, entre autres, à Dolomieu, capitaine de carabiniers et minéralogiste distingué, ou, pour la littérature, à Choderlos de Laclos, capitaine d'artillerie.

8 Observations sur la physique, sur l'histoire naturelle et sur les arts, par M. I'abbé Rozier [...] dédiées à Mgr le comte d'Artois, puis Journal de physique; cette publication mensuelle, qui accueille aussi des savants reconnus comme Lavoisier, Guyton de Morveau, Hassenfratz, Chaptal..., paraît régulièrement de 1773 à 1793.

9 Journal de physique, t. Ix, p. 379-381 (mai 1777).

10 AN Paris, T/160/4 (13 juin 1779) et T/160/5 (comité des prix, 28 décembre 1779).

11 Papers of Benjamin Franklin, New Haven et Londres, Yale University Press, vol. 31, 1995, p. 457-458 : lettre de Franklin à Servières du 8 février 1780, répondant à une lettre du 4 février ; Franklin l'éconduit poliment, prétextant ne rien connaître à la culture du tabac en Virginie. 
économiques tant dans les arts que dans la médecine des hommes et des animaux ${ }^{12}{ }^{\prime}$ et les papiers Séguier contiennent de nombreux manuscrits de lui, notamment un «Catalogue des plantes tinctoriales » (1776) et un « Catalogue des plantes acides du territoire de Nîmes » (novembre 1782). Et, en 1785, année de grande disette de fourrage, il soumet à la Société royale d'agriculture de Paris un mémoire « sur la manière de cueillir les feuilles des arbres, de les conserver et de les donner à manger aux bestiaux », dont un extrait sera imprimé en "Instruction », distribuée aux cultivateurs de la région parisienne ${ }^{13}$.

Cet intérêt manifeste pour les sciences est confirmé par un document de $1794^{14}$ dans lequel Servières, qui est alors candidat auprès du Comité de salut public pour une place « dans une administration dans les négociations ou pour la technologie raisonnée des arts \& métiers », se prévaut des travaux suivants : " II s'est appliqué à l'étude des langues mortes \& vivantes, de I'histoire \& du droit public ; des mathématiques, de la physique, de l'histoire naturelle, de la minéralogie, de la chymie, arts \& manufactures \& de l'agriculture. II a publié sur ces divers objets nombre de mémoires répandus dans plusieurs journaux. »

Son éclectisme ne se limite pas aux sciences. II s'intéresse aussi à la linguistique, participant au mouvement provençaliste qui connaît une nette accentuation au XVIII siècle. II fait le projet ambitieux d'un Dictionnaire de tous les patois méridionaux de la France, pour lequel il recueille des mots usités dans le Gévaudan et le Rouergue, se fait communiquer les travaux antérieurs de Joseph Séguier sur la langue cévenole et collationne divers dictionnaires de provençal ${ }^{15}$. Son intérêt englobe

12 « Sur la culture de l'ortie », Journal de physique, t. XIX, 1782, p. 104 et suiv., et «Mémoire pratique sur la culture de l'ortie et ses différents usages économiques », Bibliothèque physicoéconomique, instructive er amusante, année 1782, Paris, 1785, p. 1-11.

13 Mémoires d'agriculture, d'économie rurale et domestique, publiés par la Société royale d'agriculture, Paris, Buisson, 1785, p. XVI-XVII et 22-39 (mémoire lu le 17 juillet 1785) ; " M. le baron de Servières a proposé [...] différens procédés qui ont tous été répétés dans les jardins de l'École royale vétérinaire et l'expérience en a constaté l'efficacité. »

14 AN Paris, AB/XIX/5188, tableau des membres de la Société des inventions et découvertes présentant leurs titres et travaux pour postuler des emplois publics, arrêté dans sa séance du 16 nivôse an II [6 janvier 1794].

15 Bauquier Jean, notice sur Servières, Revue des langues romanes, III, 3, 1880, p. 66, et idem, Les provençalistes au XVIII' siècle, Nîmes, C. Lacour, 2003 [reprint d'articles anciens] ; Ripert E., également les belles-lettres et I'histoire : il est l'auteur $d^{\prime}$ un volume d'anecdotes sur Voltaire ${ }^{16}$ et prépare un ouvrage de révélations historiques sur la véritable identité du Masque de fer ${ }^{17}$.

Le baron de Servières paraît bien inséré dans un réseau académique provincial, particulièrement centré autour de l'Académie de marine à Brest ${ }^{18}$, de la Société royale de Montpellier, dont il est aspirant correspondant, et de I'Académie de Nîmes ${ }^{19}$. Dans la tradition de la République des lettres, il entretient des relations épistolaires avec nombre d'intellectuels, à Besançon, Montpellier (Chaptal), Nîmes (Séguier ${ }^{20}$ ), Strasbourg, et même à Genève (Sénebier) ${ }^{21}$.

Quand il quitte l'armée, Servières se retire en Gévaudan, sans qu'on sache grand-chose de son activité : « vivant mal avec son père », selon un témoignage, il donne des leçons de mathématiques aux enfants d'un

La Renaissance provençale (1800-1860), Paris-Aix-en-Provence, Champion-Dragon, 1918, reprint Marseille, Lafitte, 1978, p. 29. La Bibliothèque municipale (ensuite BM) de Rouen (Seine-Maritime) conserve plusieurs manuscrits de Servières intéressant les patois et les questions linguistiques, ainsi que les inscriptions et médailles antiques, Catalogue général des manuscrits des bibliothèques publiques de France..., Paris, Bibliothèque nationale, puis Éditions du Centre national de la recherche scientifique, 1886-1897, 48 vol., t. I, nº 1635.

16 Publiées par l'abbé Louis-Mayeul Chaudon sous le titre de Mémoires pour servir à l'histoire de M. de Voltaire, dans lesquels on trouvera divers écrits de lui peu connus sur ses différends avec J. B. Rousseau et autres gens-de-lettres, un grand nombre d'anecdotes et une notice critique de ses pièces de théâtre, Amsterdam, 1785.

17 [Attribué à Reth], Véritable clé de l'histoire de l'homme au masque de fer, Turin, 10 nivôse an XI (31 décembre 1802) ; il y annonce la publication ultérieure d'un ouvrage plus important qu'il n'aura pas le temps d'achever ; sur cet opuscule, son élaboration et sa diffusion, voir AN Paris, M/747, passim.

18 Ses archives, conservées à la bibliothèque du port de Brest, gardent trace de lettres adressées à Servières et d'un mémoire dont il est l'auteur: "Considérations sur les désavantages de l'emploi des tonneaux pour la cargaison des bâtiments et sur la nécessité de substituer des caisses quarrées », s.d., Catalogue général des manuscrits des bibliothèques publiques..., op. cit. note 15, Bibliothèques de la Marine, p. 315, 324 et 327.

19 Les papiers Séguier, à la BM de Nîmes, conservent un mémoire de Servières sur des "Observations lithologiques sur le territoire de Nîmes et lieux circonvoisins », lu à l'Académie de Nîmes le 10 avril 1783.

20 Les papiers Séguier attestent de relations régulières entre Servières et Jean-François Séguier entre 1776 et 1784, Catalogue général des manuscrits des bibliothèques publiques..., op. cit. note $15, \mathrm{t}$. VII, $\mathrm{n}^{\circ} 130$ et 230 .

21 Observations sur la physique, sur l'histoire naturelle et sur les arts, t. X, $2^{\mathrm{e}}$ semestre 1777 , p. 280-285. 
parent, le comte de Retz de Serviès, et mange « journellement » à sa table ${ }^{22}$.

Toutefois, il semble vouloir prendre part à la vie locale de sa province : ainsi, il participe aux États de Gévaudan, où il fait montre d'un grand « zèle pour tout ce qui intéresse le bien public » et défend des idées « éclairées »: en 1784, il prend publiquement position en faveur du recrutement d'un vétérinaire formé à l'école de Lyon et, en 1785, il tente de convaincre les États d'armer le clocher de la cathédrale de Mende, endommagé par la foudre, d'un paratonnerre dont il se chargerait de diriger la construction « sur les avis et le rapport de l'Académie royale des sciences $»^{23}$.

\section{5, I'année de la rupture}

La fin de I'année 1785, cependant, semble marquer un tournant décisif dans l'existence de gentilhomme provincial dilettante et émule des Lumières que mène alors le baron de Servières.

I| se lance alors dans un procès "retentissant » et calomnieux contre son parent, Pierre Jean Baptiste de Retz de Bressolles, comte de Serviès, capitaine au régiment d'Orléans-infanterie, à qui il conteste son appartenance à l'ancienne maison de Retz et le droit de porter son nom ${ }^{24}$. Il le poursuit avec une violence et un acharnement tels qu'à Mende, le scandale est grand et que son père, Urbain de Retz, doit se désolidariser de lui : « II et bien triste pour moi qu'un fils, qui auroit pu faire le bonheur de sa famille et l'avantage de ceux de son nom, s'en prenne à tous mes parents, et qu'il

22 AD Lozère, 8 J 172, Mémoire contenant griefs pour Pierre Jean Baptiste comte de Rets, ci-devant capitaine au Régiment d'Orléans, Infanterie, chevalier de l'ordre royal et militaire de Saint-Louis, seigneur de Fraissinet, [...] appellant contre Urbain Claude de Reth, baron de Servières, ancien officier, fils émancipé d'Urbain de Retz, seigneur de Servières, intimé [...], p. 8.

23 Documents relatifs à I'histoire du Gévaudan, 2e partie. Procès-verbaux des états de Gévaudan, publiés par la Société d'agriculture, sciences et arts de la Lozère, Mende, 1881, t. 8 (1780-1789), p. 159, 193 et 235 ; Journal de physique, t. 28, p. 133-135, « Lettre adressée à M. le baron de Servières par M. Boyer-Brun contenant la description d'un électroscope adaptable aux paratonnerres ». Claude-Urbain de Retz de Servières assiste aux sessions des États de Gévaudan seulement en 1784 et 1785, ce qui pourrait indiquer qu'il a quitté l'armée peu avant juin 1784 .

24 AD Lozère, 8 J 172, Mémoire contenant griefs..., op. cit. note 22, et Lescure, Armorial du Gévaudan, op. cit. note 4, p. 249, qui précise que Claude-Urbain de Retz de Servières fut débouté par le Parlement. contrarie en cela, comme EN TOUT, mes vues et les sentimens avec lesquels j'ai I'honneur d'être, Monsieur et cher cousin, votre etc. ${ }^{25}$. »

Claude-Urbain a été très tôt une source de soucis pour son père, hormis l'épisode de 1770 ; déjà, en 1776, celui-ci demandait l'aide de Séguier pour tenter de ramener son fils vers la foi :

" J'avois faits tous mes efforts pour lui inculquer [les devoirs] que la religion nous prescrits, je l'avois démontré par l'écriture sainte et prophane, et quoiqu'il eut fait ses premières communions avant son départ pour les pages en 1770, je vis avec les plus vifs regrets à son retour au bout de trois ans qu'il avoit perdu à Versailles toute croÿance ; pendant son séjour à Mende je redoublai mes efforts pour le ramener, mais je vous avoue, Monsieur, que je ne gagnai rien sur son esprit et, quoique je lui écrive de temps en temps sur cet important article, j'aÿ lieu de craindre qu'il n'a pas changé ${ }^{26}$. »

Cette fois, la mésentente devient conflit grave et son père obtient contre lui un « ordre du roi » exilant son fils à vingt lieues de la ville de Mende. Celui-ci part pour Paris.

Cet épisode semble consommer la rupture définitive entre Claude-Urbain de Retz de Servières et sa famille à tel point que, quelques années plus tard, en l'an III, quand son père demandera sa radiation de la liste des émigrés, Claude-Urbain n'interviendra absolument pas dans le traitement du dossier, pas plus que son père d'ailleurs ne fera état des fonctions officielles de son fils aîné, pourtant alors membre du Bureau de consultation des arts et métiers ${ }^{27}$. Plus encore, en 1804, au décès de Claude-Urbain, son père répondra à sa belle-fille, dans la nécessité de faire lever les scellés apposés sur ses biens, ne vouloir « en aucune manière s'immiscer dans les affaires de la succession de son fils ${ }^{28} »$ et ses autres héritiers ne lui feront aucune réponse.

On a seulement quelques d'indications sur ce que Servières fait à Paris. En 1786, il paraît avoir le projet d'ouvrir, avec un certain Boyer-Brun, une nitrière royale

25 Lettre de Servières père au comte de Retz, 14 novembre 1785, dans AD Lozère, 8 J 172, Mémoire contenant griefs..., op. cit. note 22, p. 16.

26 BM Nîmes, ms 130, lettre du baron de Servières (novembre 1776) ; je remercie ici M. François Pugnière, de I'Institut européen Séguier, qui m'a communiqué cette information.

27 AN Paris, F/7/5260.

28 AN Paris, minutier central des notaires de Paris, LIX/402, inventaire après décès de Retz-Servières ( 6 frimaire an XIII [27 novembre 1804]). 
à Nîmes, en profitant de la démolition des remparts de la ville pour en récupérer les matériaux ${ }^{29}$. À cette occasion, il intervient de la façon la plus pressante auprès de Lavoisier afin que celui-ci s'entremette pour lui procurer les autorisations nécessaires ; finalement, un différend entre les deux associés ruine le projet.

Cet intérêt particulier pour les poudres et salpêtres le conduit aussi à se livrer lui-même à des observations et à rédiger des mémoires sur la production du salpêtre : en 1786, il rappelle « à Monsieur de Lavoisier la promesse qu'il lui a faite depuis longtemps du recueil $4^{\circ}$ nouvellement imprimé sur la formation du salpêtre. Monsieur de Lavoisier n'aura point oublié que le Baron de Servières lui a communiqué beaucoup de mémoires et d'observations sur cette branche de la chimie ${ }^{30} »$.

En 1787, il fait encore des recherches approfondies sur l'ancienneté de sa maison. II cherche, dit-il, à fournir «les preuves de la cour [qui] sont aujourd'hui absolument nécessaires à quiconque veut faire son chemin dans ce païs-ci. Elles seules ouvrent la porte des grâces et de la fortune ${ }^{31}$ ». A-t-il encore en tête son procès contre le comte de Retz ? Cherche-t-il à réunir ses titres en vue d'accéder à la cour ou d'obtenir une charge officielle ? À cette époque, on le voit en effet évoluer dans l'entourage de Loménie de Brienne ; à la fin de l'année 1787, il se fait fort d'user de son crédit auprès du nouveau ministre en faveur de Roland de La Platière, en semi-disgrâce depuis la chute de Trudaine ${ }^{32}$.

En 1789, quand surviennent les élections aux États généraux, on retrouve le baron de Servières dans le cercle de Brissot, où l'a entraîné Lanthenas avec lequel il est lié depuis $1787^{33}$. II apparaît comme un observateur

29 Correspondance de Lavoisier, publiée sous les auspices du Comité Lavoisier de l'Académie des sciences, sous la dir. de Goupil M., vol. IV, 1784-1786, Paris, Belin, 1986, p. 242, 243, 248, 251, 273 et 274, correspondance entre Servières et Lavoisier (10 août-22 novembre 1786). En 1788, il reprendra contact avec Lavoisier pour procurer un débit de poudre à un de ses protégés, Correspondance de Lavoisier..., op. cit., t. V, 1787-1788, Paris, Blanchard, 1993, p. 239 (29 décembre 1788).

30 Correspondance de Lavoisier..., op. cit. note 29, t. IV, p. 242, lettre du 10 août 1786.

31 AD Lozère, 1 J 187, " mémoire de M. de Servières fils sur les titres de la maison » (1 ${ }^{\text {er }}$ juillet 1787 ).

32 Lettres de Madame Roland, publiées par Cl. Perroud, Paris, Imprimerie nationale, 1900-1902, 2 vol., t. 1, p. 673, n², et 713, n 1, et t. 2, p. 694.

33 Brissot de Warwille Jacques-Pierre, Mémoires (1754-1793), [suivi de] correspondance et papiers, publ. par Cl. Perroud, Paris, A. Picard et fils, [1912?], p. LIV et 235. attentif des événements politiques qui bientôt s'enchaînent. Un document récemment apparu en vente publique ${ }^{34}$ le montre assidu aux séances de l'Assemblée constituante ; il écoute attentivement les orateurs, note avec soin leurs propos en les assortissant parfois de commentaires, relève les réactions de la salle, les cris, les applaudissements, les interruptions ou les interpellations...

\section{La Société des inventions et découvertes}

À l'été 1790, on retrouve Servières très impliqué auprès des inventeurs et, bientôt, il est à la tête de la Société des inventions et découvertes ${ }^{35}$. Comment et pourquoi cet aristocrate en rupture en est-il arrivé à épouser la cause des artistes-inventeurs ? On est réduit aux conjectures. On trouve trace cependant, en ce qui le concerne, de relations antérieures avec des sociétés d'encouragement aux arts et métiers et à l'invention technique, nombreuses dans la seconde moitié du $\mathrm{XVIII}$ siècle ${ }^{36}$. Elles montrent que, loin d'être étranger à ce milieu, Servières y possède un réseau de connaissances. Selon le témoignage de l'abbé Mulot, il est familier des assemblées du musée de Court de Gébelin, rue Dauphine, dès 1782 ${ }^{37}$. D'ailleurs, en I'an XI, lui-même s'adresse à Moreau de Saint-Méry en qualité d'ancien " confrère du Musée de feu Gébelin ${ }^{38}$ ». II fait aussi partie, dès 1779, du public qui s'adresse à la Société libre d'émulation de l'abbé Baudeau ; la fréquentation de cette Société le conduit à faire la connaissance de I'abbé Rozier et de certains inventeurs, bénéficiaires des

34 Vente Piasa à Drouot-Richelieu (Paris), 19-20 juin 2006, $n^{\circ} 678$ : relation détaillée des débats de la Constituante pour les séances du 5 août et du 3 au 26 septembre 1789.

35 Dans un mémoire justificatif de juin 1792 (publié en annexe), Servières précise qu'il a présidé la Société des inventions et découvertes «constamment depuis le mois de janvier 1791 jusqu'au mois d'avril 1792 » (Arch. Musée des arts et métiers, NS5 [Reth]/1/2).

36 Hilaire-Pérez L., « Les sociabilités industrielles en France et en Angleterre au XVIII siècle : réseaux, institutions, enjeux », dans Benoit S., Emptoz G. et Woronoff D. éd., Encourager l'innovation en France et en Europe. Autour du bicentenaire de la Société d'encouragement pour l'industrie nationale, Paris, Éditions du CTHS, 2006, p. 201-238.

37 Tourneux M., «Journal intime de l'abbé Mulot (17771782) ", Mémoires de la Société de l'histoire de Paris et de l'îlede-France, t. XXIX, 1902, p. 19-124, p. 77-79 (24 janvier 1782) et 88-89 (31 janvier 1782).

38 AN Paris, M/747, lettre de Reth à Moreau de Saint-Méry (9 prairial an $\mathrm{xI}$. 
gratifications qu'elle distribue, comme Fortin ou Rivey, par exemple, qui seront ensuite des membres actifs de la Société des inventions et découvertes.

Servières est un homme intelligent qui comprend rapidement qu'une position à la tête de la Société des inventions et découvertes peut servir son ambition en lui ouvrant des débouchés. C'est en effet elle qui va lui permettre d'accéder à des places publiques en relation avec le monde des inventeurs et des inventions, dont le cumul va finalement faire de lui un personnage puissant ; elles vont aussi cristalliser à son encontre bien des jalousies.

Ainsi, c'est à sa situation à la tête de la Société des inventions et découvertes - et à l'action que cette dernière mène avec détermination pour I'adoption d'une législation protectrice des inventeurs, en 1790$1791^{39}$ - que Servières doit d'être nommé, à partir de juillet 1791, au Directoire des brevets d'invention.

Cet organisme, institué par la loi du 25 mai 1791 et placé sous l'autorité et la surveillance du ministre de l'Intérieur, jouit d'une relative autonomie puisqu'il est financé sur le produit de la taxe sur les brevets d'invention. II assure l'enregistrement et la transmission des brevets aux directoires départementaux, ainsi que la publicité des brevets et la collecte des sommes dues par les inventeurs ; il a également la charge de réunir les documents relatifs aux privilèges d'invention «ci-devant accordés pour des objets d'industrie ». Son rôle est essentiellement administratif : il n'a pas le pouvoir d'examiner sur le fond les demandes de brevets et de juger de leur validité puisque la loi prévoit que le brevet est accordé sur simple demande (et contre paiement d'une taxe), sans examen préalable. Cependant, dans certains cas, particulièrement quand I'inventeur demande le secret de son brevet, il peut être amené à examiner l'invention pour s'assurer de I'authenticité des descriptions déposées ${ }^{40}$. Servières semble déployer une grande activité ${ }^{41}$ dans ce poste

39 Demeulenaere-Douyère, «Inventeurs en Révolution : la Société des inventions et découvertes », op. cit. note 1 .

40 Ainsi, Servières intervient en tant que directeur des brevets dans le cas des procédés pour produire la soude industrielle déposés par Leblanc (commissaires : Servières, Darcet et Desmarest, septembre 1791) et Carny (commissaires : Servières, Parmentier et Berthollet, février 1792), Smith J. G., The origins and early development of heavy chemical industry in France, Oxford, Oxford University Press, 1979, p. 208 et 216.

41 AN Paris, F/12/997, correspondance de Servières relative aux brevets d'invention (1791-an II), et F/17/1136, p. 90-200, correspondance de Servières, directeur des brevets (10 juillet 179117 août 1792). qu'il occupe jusqu'à ce que le ministre de l'Intérieur Roland de La Platière juge bon, en octobre 1792, d'intégrer le Directoire des brevets d'invention aux bureaux de son ministère ${ }^{42}$.

C'est également la Société des inventions et découvertes qui conduit Servières au Bureau de consultation des arts et métiers : il y siège dès la constitution de celui-ci en novembre 1791 et au moins jusqu'à la fin de l'été $1794^{43}$. Contrairement au Directoire des brevets d'invention, cette instance a une fonction judicatoire officielle : véritable " jury des arts utiles», elle a pour rôle de fixer le montant des récompenses qu'elle propose au gouvernement d'attribuer aux artistes dignes d'être encouragés par la nation ; elle examine donc les mérites des inventeurs et de leurs travaux et les évalue.

Servières, qui bientôt démocratise son patronyme en Reth ${ }^{44}$, y représente la Société des inventions et découvertes, avec trois autres de ses membres. II semble $s^{\prime} y$ distinguer par une grande assiduité et jouer un rôle effectif en qualité de rapporteur, de rédacteur d'adresses ou comme chargé de divers dossiers administratifs ; il jouit même d'une certaine prééminence au sein du Bureau, où il occupe à plusieurs reprises des postes importants : il est élu secrétaire avant le 28 novembre 1792 (peut-être dès le 28 août 1792), puis vice-président en novembre 1792, président entre janvier et avril 1793, vice-secrétaire en février 1794, secrétaire le 8 mai 1794 et trésorier en juin 179445. Au printemps 1794,

42 Bernardin É., J.-M. Roland et le ministère de I'Intérieur, Paris, Société des études robespierristes, 1964, p. 238.

43 Arch. Musée des arts et métiers, 10480 (9 fructidor [an II] [25 septembre 1794]) : il annonce qu'il « part pour une mission dont il ignore le terme »; sans doute s'agit-il de sa mission en Belgique avec Wailly et Le Blond.

44 Le changement de patronyme semble systématique à partir de l'été 1794 ; le 9 fructidor an II [26 août 1794], les procèsverbaux du Bureau de consultation mentionnent « Reth dit Servières »(Arch. Musée des arts et métiers, 10480, p. 82). On peut y voir l'expression de son engagement dans la Révolution et très certainement aussi le souci d'assurer sa sécurité : certains membres de sa vaste famille et parentèle, avec lesquels il a rompu depuis 1785, ont été décrétés d'arrestation publique pour avoir pris part à la révolte du Gévaudan avec $\mathrm{M}^{\mathrm{gr}}$ de Castellane, en 1792 ; et de nombreux autres ont choisi l'émigration : ainsi, son propre frère, Auguste Guillaume Honoré, baron de Retz de Chanclos (né en 1761), sert dans la $2^{\mathrm{e}}$ compagnie à cheval des gentilshommes auvergnats de l'armée des Princes (Bodinier G., Dictionnaire des officiers de l'armée royale qui ont combattu aux États-Unis pendant la Guerre d'indépendance 1776-1783, Château de Vincennes, 1983).

45 Ballot Ch., «Procès-verbaux du Bureau de consultation des 
il signe, avec Coulomb et Hallé, le « rapport honorifique » en faveur de Lavoisier, tentative des membres du Bureau de consultation pour obtenir la libération de leur collègue qui vient d'être arrêté ${ }^{46}$.

\section{Un lourd contentieux avec Desaudray}

Le Bureau de consultation des arts et métiers focalise rapidement les critiques des artistes-inventeurs, d'autant que les manœuvres du ministre Roland pour en limiter les attributions, notamment en retardant le déblocage des crédits des récompenses, les irritent et les inquiètent.

Un des plus virulents est Charles-Emmanuel Desaudray, lui-même représentant de la Société du Point central des arts et métiers au Bureau de consultation à compter du 20 décembre 179147. De quinze ans l'aîné de Servières, il connaît bien les milieux industriels pour avoir été lui-même entrepreneur sous l'Ancien Régime. Il est l'auteur d'une véritable réflexion - plus élaborée que celle de Servières - sur le statut de l'inventeur, et publie, en mars 1792, un projet de Nouvelle constitution des sciences, arts et métiers, destinée à faire pièce aux projets trop élitistes de réorganisation de l'Académie des sciences; elle sera imprimée et largement diffusée. À la fin de cette même année 1792, il crée le Lycée des arts $^{48}$.

arts et métiers », Bulletin d'histoire économique de la Révolution, 1913, p. 15-160, passim, et Arch. Musée des arts et métiers, G 11, U 543 et V 86 ; Correspondance de Lavoisier..., op. cit. note 29, t. VII, en préparation sous la dir. de Bret Patrice, annexe VI, « Lavoisier et le Bureau de consultation des arts et métiers ».

46 Arch. Musée des arts et métiers, X 80 (4 floréal an II). 47 Ainsi, dès le 21 décembre 1791, Desaudray critique la représentation des sociétés d'artistes au sein du Bureau de consultation en déclarant «qu'il était à craindre que I'intention d'un grand nombre d'artistes ne fût de protester contre ce qui avait été fait jusqu'à présent par le Bureau, attendu que le nombre de commissaires n'ayant jamais été complet, les artistes regardaient les délibérations du Bureau comme nécessairement illégales », Ballot, "Procès-verbaux du Bureau de consultation des arts et métiers », op. cit. note 45, p. 36.

48 Hilaire-Pérez L., « Des entreprises de quincaillerie aux institutions de la technologie : I'itinéraire de Charles-Emmanuel Gaullard-Desaudray (1740-1832) », dans Belhoste J.-F., Benoit S., Chassagne S. et Mioche Ph. dir., Autour de l'industrie : histoire et patrimoine. Mélanges offerts à Denis Woronoff, Paris, Comité pour l'histoire économique et financière, 2004, p. 547567 ; Guénot H., « Une nouvelle sociabilité savante: le Lycée des arts », dans Bonnet J.-Cl. dir., La Carmagnole des Muses. L'homme de lettres et l'artiste dans la Révolution, Paris, Armand
L'antagonisme entre les deux hommes est important et Servières lui-même recueille une large part des attaques de Desaudray et de la Société du Point central. Des « libelles diffamatoires » courent sur son compte dès la fin de 1791 et le mettent en situation de devoir se défendre auprès des ministres qui se succèdent à I'Intérieur :

« Réduit à me justifier des plus horribles calomnies, il est de mon devoir, comme de mon intérêt, de prémunir la religion du ministre contre les impressions défavorables que la malveillance et la perversité s'efforcent de lui inspirer à mon égard.

Je me borne en ce moment à l'exposé sommaire des faits principaux : il donnera le fil de la plus odieuse trame qui jamais se soit ourdie contre un citoyen honnête, contre un fonctionnaire irréprochable ${ }^{49}$. »

Ce que visent ces libelles, c'est l'omniprésence - on serait tenté d'écrire l'omnipotence - de Servières à la fois à la tête du Directoire des brevets d'invention et au Bureau de consultation des arts et métiers. Ils I'attaquent sur un point particulièrement sensible dans le contexte révolutionnaire : ils mettent en cause I'honnêteté de sa gestion, avançant même l'accusation de prévarication ${ }^{50}$.

« Non content de cette attaque scandaleuse, le Sr Désaudrai, dominateur du Point central, I'électrisa si fortement qu'il y fut résolu que je serais dénoncé comme prévaricateur au ministre, à l'A[ssemblée] n[ationale] et au Roi. »

Servières se défend en mettant en avant « I'attache honorable du Comité d'agriculture et de commerce de I'Assemblée constituante, l'estime et l'amitié de $\mathrm{M}^{r}$ de Boufflers, enfin les bontés de $M^{r}$ de Lessart », qui ne sont que les justes récompenses « des pénibles travaux et des sacrifices [qu'il a], toute [sa] vie, faits à l'amour des arts», et surtout la confiance que lui témoigne « $\mathrm{M}^{\mathrm{r}}$ Blondel, vice-président du Comité central du commerce ». L'attaque étant la meilleure des défen-

Colin, 1988, p. 67-78.

49 Arch. Musée des arts et métiers, NS5 [Reth]/1 (2), mémoire justificatif de Servières du 30 juin 1792, publié intégralement en annexe.

50 Cette accusation le poursuivra longtemps, notamment en 1801, alors qu'il est à Turin pour mettre en place la Loterie nationale : s'il assure avoir rendu, au cours de l'été 1792, le compte des deniers reçus par lui comme directeur des brevets et que ce compte a été accepté, les scellés sont néanmoins mis sur son domicile parisien et un gardien désigné pour ses biens (AN Paris, $A J / 14 / 188)$. Et, effectivement, si on se réfère à sa succession, on ne peut guère le suspecter d'enrichissement abusif. 
ses, Servières dénonce à son tour la Société du Point central comme une " société turbulente et sans choix, qui [...] a toujours porté envie à celle des Inventions et découvertes», et son leader, le " Sr Gaulard-Désaudrai, qui fut refusé [à la Société des inventions et découvertes] au mois de septembre [1791], à cause de son immoralité publiquement reconnue ». Les accusations de Desaudray ne seraient donc dictées que par le dépit et la jalousie...

Desaudray accuse également Servières d'avoir tenté d'user de son influence pour discréditer la Société du Point central et l'éliminer en faisant admettre au Bureau de consultation des arts et métiers, pour l'y représenter, deux inventeurs, Calippe et Droz ${ }^{51}$, qui n'étaient pas membres du Point central.

Quelle est la réalité de ces accusations ? Au Bureau de consultation, Servières a-t-il, en signant des rapports favorables, plutôt avantagé des inventeurs membres de sa société au détriment des autres ? Au Directoire des brevets, a-t-il fait supporter des « longueurs injustes » à certains membres du Point central, comme au moins sept d'entre eux, dont Desaudray, l'affirment ? Faut-il croire les allégations d'un de ses anciens collaborateurs qui, « indigné de voir les vexations et abus de confiance de $M$. de Servières envers les artistes, aima mieux quitter une meilleure place qu'il avait au Bureau des brevets, et en prendre une moindre [...] pour ne pas être compromis dans les écarts odieux qu'il voyait commettre aud. $\mathrm{S}^{r}$ de Servières ${ }^{52} »$ ?

Le contentieux entre les deux hommes est lourd. Derrière les attaques personnelles et la surenchère à laquelle ils se livrent, se dessine un enjeu financier d'importance : en quatre années d'activité, le Bureau de consultation des arts et métiers distribue plus d'un million de livres à environ trois cents artistes ${ }^{53}$... de quoi se faire beaucoup d'obligés, d'autant que pour nombre $d^{\prime}$ artistes-inventeurs il en va de leur survie économi-

51 Ils seront finalement remplacés par Desaudray et Dumas, le 20 décembre 1791 (Arch. Musée des arts et métiers, 10³92, « Point central des arts et métiers »).

52 Ibid.

53 Place D. de, «Le Bureau de consultation pour les Arts, Paris, 1791-1796», History and technology, 5, 1988, p. 139-178, p. 140. Une anecdote souligne à quel point les membres de la Société des inventions et découvertes sont intéressés au fonctionnement régulier du Bureau de consultation des arts et métiers : pendant le rigoureux hiver de I'an III (1794-1795), ils lui font l'avance d'une voie et demie de bois de chauffage pour lui éviter d'avoir à interrompre ses travaux (ibid., p. 146). $q^{2} e^{54}$. L'enjeu financier est donc finalement un enjeu de pouvoir.

Servières a beau crier au complot et protester de son honnêteté, ces querelles, fondées ou non, sèment la discorde et contribuent à affaiblir le poids politique des artistes-inventeurs qui se divisent ; certains se retrouvent dans une situation ambiguë.

La discorde qui s'affirme entre les deux sociétés d'inventeurs, contribue aussi sans aucun doute à ruiner l'influence de la Société des inventions et découvertes que Servières préside jusqu'en avril 1792, et aussi celle de Servières lui-même. Avec sa Nouvelle constitution des sciences, arts et métiers, c'est son adversaire, Desaudray, qui, à la tête du Point central des arts et métiers, en tirera avantage pour prendre le leadership des inventeurs.

\section{Des assignats à la Loterie nationale}

En juillet 1793, Claude Urbain Reth-Servières, « domicilié rue l'Évêque 1, section Butte des moulins », se retrouve mêlé à une affaire qui aurait pu lui coûter très cher, et qui montre à quel point il a contre lui des adversaires déterminés, même s'il est difficile de démêler la part qu'y prennent les artistes-inventeurs mécontents et celle d'autres ennemis éventuels. II dépose au Comité de Sûreté générale une correspondance adressée, sous son couvert et à son insu, au député Charles Barbaroux par un nommé Couédic. II raconte qu'il a connu ce dernier en mars 1793 chez Barbaroux, auquel le député à la Convention Pocholle l'avait engagé à s'adresser pour lui communiquer les cartes et plans du projet de canal de Paris à Dieppe du Citoyen Le Moyne - Reth se présente alors comme « ingénieur-géographe ». II manifeste avec vigueur sa surprise et son indignation de ce que l'on s'obstine à vouloir le rendre colporteur d'une correspondance, « criminelle » à ses yeux, qui se réjouit de la mort de Marat et affirme des opinions fédéralistes, « diamétralement opposées à ses principes ». II y a là indéniablement, sans qu'on sache d'où vient le coup, tentative pour le compromettre et l'entraîner dans la chute des Girondins, un piège dont, sans état d'âme, il se dégage au prix d'une dénonciation ${ }^{55}$.

54 Nombreux sont les artistes-inventeurs qui se plaignent, à juste titre ou non, de s'être ruinés pour développer leurs inventions ; on peut citer notamment Jean-François Gavoty, introducteur de la sparterie en Europe, ou François Cointereaux, inventeur de la construction en pisé.

55 AN Paris, F/7/4585, dossier Barbaroux; Chabaux A., « Un 
Servières est toujours très actif, au point même parfois d'en paraître brouillon. Ainsi, on le voit employé à diverses missions, comme au début de 1795 où il accompagne en Belgique, en qualité de secrétaire, les représentants en mission Wailly et Le Blond ${ }^{56}$ pour choisir des objets de sciences et arts destinés à enrichir les musées et bibliothèques de la République française.

Bientôt, on le retrouve à la tête de la fabrication des assignats. En mai 1795, il est nommé directeur des artistes des assignats ${ }^{57}$. La question est loin de lui être étrangère, car, au début de 1793, il a été désigné, avec Lavoisier, Trouville, Jumelin, Desmarest et Berthollet, pour constituer une commission du Bureau de consultation des arts et métiers chargée de perfectionner la fabrication des assignats et d'en prévenir la contrefaçon ${ }^{58}$, et c'est sans doute cette expérience qui lui confère la légitimité nécessaire pour cet emploi.

En fait, il arrive assez tardivement dans I'histoire des assignats. Son rôle va être surtout de liquider I'entreprise. La loi du 9 pluviôse an IV [29 janvier 1796] ordonne en effet de briser «les formes, planches et matrices, les poinçons, signes caractéristiques et ustensiles qui ont servi ou dû servir à la fabrication des

collaborateur de Barbaroux, I'ingénieur du Couédic », Annales historiques de la Révolution française, 1935, p. 249-253.

56 Charles Wailly ou Dewailly, architecte, et Gaspard Michel, dit Le Blond, archéologue; le 4 avril, ce dernier présente Reth à la Commission " comme ayant rendu des services essentiels aux arts, en contribuant autant qu'il était en lui au succès des recherches et à l'exécution des vues des commissaires artistes ", Tuetey L. Procès-verbaux de la Commission temporaire des arts, Paris, Imprimerie nationale, 1912, t. 2, p. 166 (10 mars 1795) et p. 198 (4 avril 1795).

57 Mercier A., L'argent des révolutionnaires, exposition du musée national des Techniques, Paris, 1989, p. 11 et suiv. Outre Reth, Groizier est directeur des imprimeries et Grouvelle directeur des timbres, tous trois avec des appointements de 6000 livres, AN Paris, AF/III/29, p. 56 et 57 (nivôse an IV).

58 Lavoisier l'indique dans une demande d'attestation de services le concernant adressée au Bureau de consultation des arts et métiers (Euvres de Lavoisier, éd. par J.-B. Dumas, É. Grimaux et F.-A. Fouqué, Paris, Imprimerie impériale, 1862-1893, t. IV, p. 714). Servières paraît avoir joué un rôle actif à ses côtés dans cette commission (ibid., p. 699). II semble même qu'on lui reconnaisse - ou qu'il cherche à se faire reconnaître - une certaine expertise dans la lutte contre la contrefaçon des papiers publics ; ainsi, en messidor an VI, il rédigera, sous le nom de " Reth, exdirecteur du papier-monnaie », avec l'ancien conventionnel Bonet de la Haute-Loire, un Mémoire théorique et pratique sur les moyens d'assurer la police des passeports dans toute la République, imprimé par le Conseil des Cinq-Cents en pluviôse an VII. Sans doute est-il alors à la recherche d'un nouvel emploi public et cherche-t-il à faire valoir ses compétences. assignats, en exécution de la loi du 2 nivôse an IV [23 décembre 1795] mettant fin à l'émission des assignats et prévoit la destruction du matériel », et, le 30 pluviôse an IV [19 février 1796], a lieu place Vendôme, à Paris, un grand autodafé symbolique au cours duquel la plus grande partie du matériel de fabrication des assignats est brisé et brûlé ; le reste du matériel est vendu aux enchères en novembre 1796. II faut néanmoins saluer le souci de Servières-Reth de préserver le patrimoine technique lié aux assignats : il demande au secrétaire du Conservatoire national des arts et métiers, Molard, de recueillir certains objets-témoins de la fabrication des assignats et signe avec Grouvelle et Groizier, le 19 brumaire an V [9 novembre 1796], un « mémoire sur la nécessité de conserver, dans un établissement public, le grand balancier de la fabrication du papiermonnaie ${ }^{59} »$.

En brumaire de l'an VI [novembre 1797], en reprenant cette fois le nom de Servières, peut-être pour pouvoir se prévaloir de ses travaux scientifiques publiés sous ce nom avant 1789, il est un des douze candidats au remplacement de Lazare Carnot à la Section de mécanique de l'Institut national des sciences et des arts, en concurrence avec un certain général Bonaparte qui, comme on sait, l'emportera ${ }^{60}$.

Dès lors, le ci-devant baron de Servières, aristocrate en rupture, se mue définitivement en Reth, bureaucrate bourgeois acquis au nouveau régime, qui proteste à qui veut l'entendre de son attachement aux principes républicains.

À l'issue de l'épisode des assignats, jamais à court d'expédients et sans doute en faisant jouer ses relations ${ }^{61}$, Reth se « recase » à la Loterie nationale, met-

59 Cette proposition sera adoptée par le Conseil des Cinq-Cents le 21 brumaire an V [11 novembre 1796].

60 Procès-verbaux des séances de l'Académie tenues depuis la fondation de I'Institut de France jusqu'au mois d'août 1835, Paris, 1910, t. 1, p. 296, séance du 21 brumaire an VI [11 novembre 1797]: "La section de mécanique présente pour le remplacement du $C^{n}$ Carnot une liste de douze candidats, qui sont les C ns Buonaparte, Montalembert, Lamblardie, Dillon, Louis Berthoud, Bréguet, Janvier, Callet, Grobert, Molard, Lenoir et Servières. » Le scrutin donne les résultats suivants : Buonaparte : 411 voix, Dillon: 371, Montalembert : 367, Lamblardie : 348, Molard : 303, Louis Berthoud : 267, Callet : 265, Bréguet : 206, Lenoir : 191, Janvier : 157, Grobert : 124 et Servières : 106. » Bonaparte, Dillon et Montalembert sont proposés à l'assemblée générale de I'Institut qui choisira le premier le 5 nivôse an VI [25 décembre 1797].

61 Vraisemblablement grâce à la protection du ministre des Finances Gaudin qu'il a connu alors que celui-ci était commissaire 
tant à profit la re-création de cette dernière par le Directoire en septembre 1797. En 1798, I'Almanach national le mentionne comme l'un des trois chefs de la « Section des départements » de la division de la correspondance générale de la Loterie nationale ; il semble être inspecteur à Bordeaux, puis, en 1802, il passe chef de division de la correspondance générale, des dépêches et du secrétariat, à Paris.

Cependant, les Français occupent le Piémont et y mettent en place une nouvelle administration. L'arrêté du 19 fructidor an IX [6 septembre 1801] remplace le Lotto del Seminario par la Loterie nationale de France ; son article 5 prescrit l'envoi à Turin d'un inspecteur « pour faire de concert avec le Commissaire extraordinaire français chargé de l'organisation des finances et I'Administrateur général [...] toutes les dispositions nécessaires pour organiser l'établissement de la Loterie nationale selon les lois et règlements français, et pour y établir le nombre de bureaux qui seront jugés convenables ${ }^{62} » . C^{\prime}$ 'est le « Citoyen Reth, chef de division de la Correspondance générale de l'administration de la Loterie nationale », qui est chargé de cette mission spéciale. Désigné le 2 vendémiaire an X [24 septembre 1801], il quitte Paris dès le 5 pour arriver à Turin le 20 vendémiaire ${ }^{63}$ et se mettre aussitôt à l'ouvrage.

Organiser la loterie nationale à Turin et dans les six départements qui constituent le Piémont, semble être une tâche dont Reth s'acquitte avec le zèle et l'activité qui lui sont habituels, ainsi que l'attestent ses abondantes archives ${ }^{64}$. Mais peut-être se laisse-t-il aller à quelques imprudences. À Paris circulent bientôt contre lui des rumeurs qui visent son train de vie dispendieux et peut-être ses irrégularités de gestion ; quelle que soit la réalité de ces allégations, elles provoquent sa suspension brutale le 22 germinal an XI [12 avril 1803].

Durant son séjour italien, aussi occupé soit-il par ses tâches administratives, Reth ne renonce pas à son

à la Trésorerie nationale et que lui-même dirigeait la fabrication du papier-monnaie, AN Paris, M/747, lettre à Gaudin du 23 nivôse an XI [13 janvier 1803].

62 Bulletin des actes de l'Administration générale de la $27^{e}$ division militaire, $\mathrm{n}^{\circ} 40,3$ brumaire an $X$ [25 octobre 1801].

63 AN Paris, AJ/14/180, passim.

64 Les archives de la Loterie de Turin, conservées aux Archives nationales (site de Paris), en AJ/14/179 à 188, comportent de nombreux dossiers administratifs relatifs à la mise en place des différents rouages de la loterie et une innombrable correspondance de Reth, tant de fonction (avec les administrateurs de la Loterie à Paris, notamment) que personnelle (lettres de sa femme et d'amis et relations diverses). insatiable curiosité. II trouve encore le temps de faire des recherches dans les bibliothèques pour réunir une documentation abondante sur la véritable identité du Masque de fer, question qui le passionne et sur laquelle il prépare un ouvrage historique.

Reth rentre donc précipitamment à Paris. II y retrouve ses fonctions de chef de bureau à la Loterie nationale et on n'entend plus parler de lui jusqu'à son décès qui survient le 27 octobre 1804, 10 rue Neuve des Petits Champs ${ }^{65}$. Sa succession est modeste : ne comportant que son mobilier, son cautionnement et les appointements à percevoir à son décès, elle se monte à 4 879,52 francs. Finalement, sa veuve, " commune en biens avec lui sans contrat de mariage », renonce à la succession et c'est son père, Urbain de Retz, et ses frère et sœurs survivants qui en bénéficient. Sa disparition laisse encore des interrogations en suspens, tant est difficile à saisir le personnage : ainsi, sur sa situation matrimoniale réelle ${ }^{66}$ ou sa fortune ${ }^{67}$ et même sur les circonstances de l'entrée de ses papiers aux Archives impériales en $1809^{68} \ldots$

\section{Conclusion}

Authentique aristocrate formé aux Lumières, puis entré en rupture avec son milieu, passé à la Révolution sans doute autant par opportunisme que par esprit de modernisme, le baron de Servières a finalement joué,

65 Arch. Paris, D.Q/8/17, tables de décès et d'absences de l'Enregistrement, $8^{\mathrm{e}}$ bureau (Paris, $1^{\mathrm{er}}$ et $2^{\mathrm{e}}$ arrondissements anciens). 66 À son décès, les documents officiels le donnent comme marié à Marie Jeanne Adélaïde Duguet, alors que les «lettres de ma femme », conservées sous la cote AN Paris, AJ/14/188, sont signées « Éléonore »; il semble par ailleurs avoir eu une vie sentimentale un peu embrouillée.

67 Arch. Paris, D.Q/7/1712, et AN Paris, minutier central des notaires de Paris, ét. LIX/402, inventaire après décès, 6 frimaire an XIII [27 novembre 1804].

68 L'introduction de l'inventaire de la sous-série G/4 des Archives nationales (Archives nationales, Archives du bureau de la loterie nationale. Inventaire de la sous-série G/4, établi en 1894 par René Teulet, revu et complété), indique que les services de la Direction générale de la liquidation rapatrient, en juillet 1809, à la fois les archives du bureau de la loterie de Turin (auj. soussérie $\mathrm{AJ} / 14)$ et les papiers "littéraires» de Reth $(M / 747)$. Une note accompagnant ces derniers indique qu'ils ont été « trouvés dans les malles et effets mis à la Messagerie et non retirés », et précise que le dépôt fait aux Archives impériales se composait de quatorze cartons de papiers, d'une pièce d'or de Marengo de 20 francs, de monnaies de Savoie en argent, de diverses médailles d'argent, bronze et autres métaux, de livres et de brochures (AN Paris, M/747). 
son ambition personnelle aidant, un rôle important dans I'histoire de l'invention en France. À la tête de la Société des inventions et découvertes, dont il a su orchestrer habilement I'action, souvent à son propre avantage, il a obtenu le vote des lois fondamentales qui ont établi le statut de l'invention en France. Sans avoir, comme Desaudray, la prétention d'une réflexion de fond sur ces questions, il a néanmoins pris une part non négligeable dans l'institutionnalisation de l'invention.

Si la suite de son itinéraire - administration des Assignats, puis de la Loterie nationale - est partagée entre engagement réel au service des principes républicains et remarquable faculté à rebondir, elle semble moins heureuse et moins propre à satisfaire cet insatiable, curieux de tout, toujours à l'affût d'un « coup».

Alors, providence des inventeurs ou vulgaire arriviste ? Républicain convaincu ou déclassé aux abois ? Ces questions résument toute la complexité du personnage et l'originalité de sa démarche. Mais Claude-Urbain de Retz de Servières, dit Reth, mérite certainement mieux que le semi-incognito dans lequel il est resté.

\section{Annexe \\ La querelle Servières-Desaudray}

Compte rendu à Monsieur Cahier de Gerville,ministre de l'Intérieur, par $\mathrm{M}^{r}$ de Servières, pour détruire les inculpations et les calomnies de la Société du Point central des arts \& métiers, 5 décembre 1791 (AN Paris, $A B / X I X / 5188)$.

"Monsieur,

Sur ce que vous m'avez fait I'honneur de me dire, ce matin, qu'une députation de la Société du Point central étoit venüe se plaindre à vous contre moi, j'ai pris l'engagement de vous démontrer mon innocence $\&$ l'injustice de telles plaintes. Vous m'avez offert la communication des pièces : en cela j'ai reconnu l'esprit d'impartialité qui vous anime ; mais je n'ai pas besoin de ces pièces qui ne sont qu'un tissu d'assertions hazardées \& mensongères. La fidélité de ma mémoire \& l'évidence de ma cause suffiront à ma défense.

La marche que je vais suivre sera droite \& franche comme la vérité. Je me bornerai donc à mettre sous vos yeux une suite de faits, que j'ai racontés, le mercredi 30 gre [novembre], au Bureau de consultation pour les arts \& métiers, \& que j'ai exposés, le lendemain jeudi $1^{\text {er }} X^{\text {bre }}$ [décembre], dans le même ordre, à la Société nationale des inventions \& découvertes, séante au Louvre.

$M^{r}$ de Boufflers sur le rapport duquel avoient été rendus les décrets des $9 \& 10$ septembre, qui fixent une somme annuelle de 300 mille livres pour les récompenses nationales aux artistes, \& celui du 27 du même mois de septembre, relatif à la formation d'un Bureau de consultation pour l'examen des demandes \& la distribution des récompenses, me chargea, en partant de Paris, au mois d'octobre, de proposer à $\mathrm{M}^{r}$ Delessart le Sr Calippe comme un artiste digne par ses talens d'être membre de ce bureau.

En effet, je m'acquittai de cette mission de confiance auprès de $\mathrm{M}^{r}$ Delessart, qui me répondit avoir déjà reçu un grand nombre de semblables demandes, \& m'autorisa néanmoins à lui reparler du Sr Calippe.

[en marge : 24] Je le fis le 24 octobre, en présentant au ministre le projet de lettre qu'il m'avoit demandé pour inviter l'Académie des sciences à s'occuper [document incomplet].

Servières à Cahier de Gerville, ministre de I'Intérieur, 6 décembre 1791, minute (Arch. Musée des arts et métiers, NS5 [Reth]/1/1).

Vous ayant exposé la répugnance bien fondée que j'éprouve à demander la communication du libelle diffamatoire que la Société du Point central vous a fait passer contre moi, à la personne que vous m'avez nommée, je vous prie et j'espère de votre équité que vous aurez la bonté de me faire passer directement les pièces. II est nécessaire que je puisse en prendre lecture, avant de vous envoyer mon mémoire, afin qu'il ne manque rien à ma défense et que la calomnie soit confondue.

J'ai I'honneur etc. etc.

Blondel, vice-président du Comité central du commerce, à Cahier de Gerville, ministre de I'Intérieur, 13 mars 1792, copie (AN Paris, F/12*/140, fol. 19).

M.,

J'ai lu, avec attention, la lettre que vous m'avez fait l'honneur de me communiquer et qui contient des plaintes sur la conduite de M. de Servières, directeur du Bureau des brevets d'invention. La conversation que le $S^{r}$ Defoy, auteur de cette lettre, rapporte et qu'il prétend avoir été tenue par les commis de M. de Servières, m'a donné occasion de vérifier ce fait. J'ai en 
conséquence entendu, séparément, trois de ces emploïés (les $s^{\text {rs }}$ Huart, Mathieu et Bicheret) qui viennent exactement matin et soir à leur bureau. Mais ils m'ont assuré qu'ils n'avoient aucune connaissance de cette prétendue conversation, qu'elle n'avoit point été tenue en leur présence, et qu'ils n'avoient aucun sujet de se plaindre de M. de Servières. II seroit donc possible que les inculpations dirigées contre ce dernier ne fussent pas exactes, et ce qui me porte à croire qu'elles ne sont que l'effet de quelque haine particulière contre lui, c'est que la lettre du S'Defoy ne paraît pas avoir été écrite par lui et qu'il n'a fait que la signer.

Je ne prétends cependant pas, M., justifier M. de Servières dans sa conduite privée que je ne connois pas. J'aurai seulement l'honneur de vous observer que je ne l'ai jamais vu ivre et qu'il me paroît mettre l'activité nécessaire dans le travail dont il est chargé. Je suis avec respect etc.

Mémoire justificatif de Servières, 30 juin 1792 (Arch. Musée des arts et métiers, NS5 [Reth]/1/2).

Réduit à me justifier des plus horribles calomnies, il est de mon devoir, comme de mon intérêt, de prémunir la religion du ministre contre les impressions défavorables que la malveillance et la perversité s'efforcent de lui inspirer à mon égard.

Je me borne en ce moment à l'exposé sommaire des faits principaux : il donnera le fil de la plus odieuse trame qui jamais se soit ourdie contre un citoyen honnête, contre un fonctionnaire irréprochable.

La notoriété publique atteste que c'est à mes soins, à mon zèle, à mon activité que sont dus deux établissements, d'abord reçus avec transport et qui doivent exercer la plus prompte et la plus heureuse influence sur le développement de l'industrie nationale. Ces établissements sont le Directoire des brevets d'invention et le Bureau de consultation des arts et métiers. Le premier existe d'après les lois du 7 janvier et 25 mars 1791 ; le second, d'après celle du $127^{\text {bre }}$ [septembre] et 16 octobre.

Le vœu unanime des artistes, I'attache honorable du Comité d'agriculture et de commerce de I'Assemblée constituante, l'estime et l'amitié de $\mathrm{M}^{\mathrm{r}}$ de Boufflers, enfin les bontés de $\mathrm{M}^{r}$ de Lessart, pour prix d'une année entière de pénibles travaux et des sacrifices que j'ai, toute ma vie, faits à l'amour des arts, me placèrent, au commencement de juillet 1791, à la tête du Directoire des brevets d'invention.

Au mois de novembre suivant, une société respectable, formée depuis le mois de janvier et connue sous le nom des Inventions et découvertes, me choisit, à une grande majorité, le premier des quatre députés que $\mathrm{Mr}^{r}$ Delessart avoit bien voulu lui accorder au Bureau de consultation ; et j'y pris séance le 19, jour de son ouverture.

Dans les derniers jours de ce même mois de novembre, $\mathrm{M}^{r}$ Delessart remit le ministère de l'Intérieur à $\mathrm{M}^{\mathrm{r}}$ Cahier de Gerville.

Celuy-ci avoit pour confident et pour ami un $\mathrm{M}^{\mathrm{r}}$ Lalouette.

Il existoit une liaison, dont j'ignore l'origine, entre ce $\mathrm{Mr}^{r}$ Lalouette et le $\mathrm{S}$ r Boisseau, mon premier commis, homme avide, violent et faux, lequel ne pouvoit me pardonner de n'avoir point eu ma place, qu'il avoit convoitée du moment que l'on s'occupa de la créer.

Dès les premiers jours de décembre, ce $\mathrm{S}$ r Boisseau, par la funeste entremise de $\mathrm{M}^{r}$ Lalouette, fit remettre au ministre des mémoires affreux contre moi. Lui-même en distribua avec profusion à $\mathrm{M}^{r}$ Blondel, vice-président du Comité central du commerce, au Directoire du département de Paris, à $\mathrm{M}^{r}$ Tarbé, ministre des Contributions, $\mathrm{M}^{\mathrm{r}}$ Lavoisier et à d'autres membres du Bureau de consultation etc. etc.

Mon travail n'étant plus direct avec le ministre, celuy-ci ne m'ayant donné communication d'aucune de ces pièces diffamatoires, il me fut bien impossible de me justifier. Aussi, les préventions germèrent tellement dans l'esprit de $\mathrm{M}^{\mathrm{r}}$ Cahier que sans examen et sans motif il vouloit me dépouiller d'une place, fruit de mes sueurs, pour en revêtir un de ses parens. La droiture et les bontés de $\mathrm{M}^{r}$ Blondel qui connoissoit toute la noirceur de l'intrigue du Sr Boisseau, me sauvèrent alors de cette flétrissante injustice, qui m'auroit ravi tout ensemble I'honneur et mon état.

Démasqué, furieux de n'avoir pu consommer ma ruine et certain qu'il avoit perdu sans retour ma confiance, le Sr Boisseau sentit qu'il étoit tems de se retirer. II négocia donc l'échange de sa place avec le $\mathrm{S}$ r Rémy, commis au secrétariat du Département de Paris. Les conventions secrettes une fois arrêtées entre eux, le $S^{r}$ Boisseau donna sa démission, et par une décision de Mr Roland, du 26 mars, le Sr Rémy vint le remplacer dans mon bureau ${ }^{69}$.

Depuis qu'il est au département, le Sr Boisseau n'a cessé de me diffamer avec tout l'acharnement de la scélératesse ; et il provoque journellement les artistes à se coaliser avec lui pour me perdre. C'est de là qu'il

69 AN Paris, F/12*/140, fol. $21 r^{\circ}$, la copie de la lettre de Blondel du 29 mars 1792 confirme ces informations. 
a l'audace d'écrire à ses complices que par délicatesse il a sacrifié un emploi considérable pour un moindre, afin de n'être plus témoin des vexations, des abus de confiance et des concussions qu'il me voyoit commettre.

Ici, j'oppose, avec une pleine sécurité, aux atroces calomnies du Sr Boisseau, le témoignage irrécusable de Mr Blondel et du Comité central de commerce, auxquels j'ai rendu les comptes les plus fidèles de ma gestion. Elle a été reconnue pure, loyale et désintéressée.

Maintenant, il me reste à faire connoître les motifs de l'animosité que depuis six mois la Société du Point central des arts et métiers fait éclatter contre moi.

Cette société turbulente et sans choix, qui ne s'est formée qu'au mois d'avril ou de may 1791, a toujours porté envie à celle des Inventions et découvertes, que j'ai eu l'honneur de présider constamment depuis le mois de janvier 1791 jusqu'au mois d'avril 1792.

Plusieurs membres du Point central, faute par eux de satisfaire à ce qu'exigeoit le règlement de la Société des inventions et découvertes, n'ont pu y être admis. Inde iroe ! Toute la haine de la médiocrité s'est concentrée au-dessus de ma tête.

Au nombre des candidats ainsi exclus, se trouve un Sr Gaulard-Désaudrai, qui fut refusé au mois de septembre, à cause de son immoralité publiquement reconnue. II jura dès lors ma perte; et depuis cette époque il n'a cessé de la machiner, en faisant jouer tous les ressorts des plus viles intrigues. Des libelles odieux ont été répandus, où il me diffame, sans oser me nommer.

Membre et meneur du Point Central, le $S^{r}$ Désaudrai souleva facilement contre moi cette société qui envoya au mois de décembre des députations au ministre et au Bureau de consultation, pour m'en faire exclure ; mais ces plaintes étoient si mal fondées que je suis demeuré inébranlable à mon poste.

Une différence d'opinion au sujet de récompense pour le $\mathrm{S}^{r}$ Rivey et pour le Sr Michel m'attira, dans le mois de février et de mars, les outrages les plus sanglans de la part du Sr Désaudrai, en présence du Bureau de consultation, où l'intrigue seule l'avoit fait admettre.

Non content de cette attaque scandaleuse, le Sr Désaudrai, dominateur du Point central, I'électrisa si for- tement qu'il y fut résolu que je serais dénoncé comme prévaricateur au ministre, à l'A[ssemblée] n[ationale] et au Roi.

Par une lettre détaillée du 20 mars, j'instruisis de ce complot infernal $\mathrm{M}^{r}$ Blondel, qui eut la bonté de me tranquilliser, en m'assurant, comme il me l'a répété bien des fois, qu'il ne souffriroit pas qu'il me fût fait aucune injustice.

Presque toutes les séances du Point central n'ont été occupées que de ma diffamation et qu'à combiner les moyens de rendre ma perte inévitable. Malgré les récla[ma]tions de quelques membres, il a été décidé que l'on ne me donneroit communication d'aucune des plaintes suscitées contre moi, afin de me mettre hors d'état de défense. On a voulu m'attaquer à l'improviste.

La dénonciation au ministre n'a été différée que parce que les agitateurs du Point central, sachant que j'étois connu, aimé et j'ose le dire estimé de $\mathrm{M}^{r}$ Roland, ils ont senti qu'il leur seroit fort difficile de me détruire dans son esprit.

Enfin, le 26 de ce mois, les députés de cette société dénonciatrice ont remis à Monsieur Terrier une adresse diffamatoire, qui ne se trouve appuyée d'aucune pièce probante.

Le ministre a pris le parti que lui dictoient la prudence et la justice. Il a renvoyé l'examen de cette affaire au Bureau de consultation. Je me suis, avec une aveugle confiance, soumis au jugement de ce tribunal, composé d'hommes aussi honnêtes qu'intègres et éclairés.

En attendant que d'après la communication des pièces qui ont été demandées, je puisse rédiger et fournir ma défense au Bureau, d'après l'avis duquel le ministre prononcera sur mon sort, je supplie le Comité central de commerce et particulièrement $\mathrm{M}^{r}$ Blondel de m'accorder leur appui auprès de Monsieur Terrier, et d'obtenir de son impartialité qu'il s'abstienne de prendre aucune opinion jusques à ce que l'affaire soit parfaitement instruite et que le Bureau de consultation ait pu lui donner à cet égard son avis motivé. Un intervalle immense sépare l'accusation de la conviction, et ma conscience me dit que je dois sortir triomphant du combat à mort qui m'est livré par la rage et l'envie.

À Paris, le 30 juin 1792, I'an 4' de la Liberté. 